\title{
iHDI 2020: Interdisciplinary Workshop on Human-Drone Interaction
}

\author{
Mehmet Aydın Baytaş \\ Koç University \\ Istanbul, Turkey \\ mbaytas@ku.edu.tr \\ Markus Funk \\ Cerence Inc. \\ Ulm, Germany \\ markus.funk@cerence.com \\ Sara Ljungblad \\ University of Gothenburg \\ Chalmers University of \\ Technology \\ Gothenburg, Sweden \\ sara.ljungblad@gu.se \\ Jérémie Garcia \\ ENAC, Université Toulouse \\ Toulouse, France \\ jeremie.garcia@enac.fr \\ Joseph La Delfa \\ Monash University \\ RMIT University \\ Melbourne, Australia \\ joseph@exertiongameslab.org \\ Florian 'Floyd' Mueller \\ Monash University \\ RMIT University \\ Melbourne, Australia \\ floyd@exertiongameslab.org \\ Permission to make digital or hard copies of part or all of this work for personal or

\begin{abstract}
Human-drone interaction (HDI) is becoming a ubiquitous topic in daily life, and a rising research topic within $\mathrm{CHI}$. Knowledge from a wealth of disciplines - design, engineering, social sciences, and humanities - can inform the design and scholarship of HDI, and interdisciplinary communication is essential to this end. The Interdisciplinary Workshop on Human-Drone Interaction (iHDI 2020) aims to bring together diverse perspectives; advancing $\mathrm{HDI}$ and its scholarship through a rich variety of activities involving an assortment of research, design, and prototyping methods. The workshop intends to serve as a platform for a diverse community that continuously builds on each other's methods and philosophies, towards results that "take off."
\end{abstract}

\section{Author Keywords}

Drones; unmanned aerial vehicles (UAVs); flying user interfaces; human-drone interaction; quadcopters.

\section{CCS Concepts}

-Human-centered computing $\rightarrow$ Interaction paradigms; Interaction devices; $\mathrm{HCl}$ theory, concepts and models; User interface design; 


\section{Past Related Workshops}

iHDI: First International Workshop on Human-Drone Interaction at ACM CHI $2019[7]$

hdi.famnit.upr.si

Human-Robot Interaction with UAVs: Challenges and Frontiers at IEEE ICRA 2018

iri.upc.edu/workshops/hriuav18

Human-Robot Interaction for Small and Personal Unmanned Aerial Vehicles at RSS 2016

cse.unl.edu/ bduncan/rss/

\section{Background}

Flying drones are poised to become ubiquitous [2, 11]. Already they are common in a variety of research and practical applications including construction, emergency operations, logistics, accessibility, and exercise. Drones are also emerging as tangible user interface components in $\mathrm{HCl}$ applications [1, 13, 15]. The significance of human-drone interaction (HDI) as a topic of interest within the $\mathrm{CHI}$ community was also evidenced by a full session focusing on drones at the $\mathrm{CHI}$ '19 main track [2, 9, 14, 26].

Current HDI research field builds on a diverse motivations and methodologies, with contributions originating from various clusters worldwide. As HDI researchers in the $\mathrm{CHI}$ community, we observe significant potentials for expediting and compounding research efforts where different research efforts intersect. Thus we propose to hold an interdisciplinary and hands-on HDI workshop with participants from an expansive variety of disciplines, including engineering, design, and humanities. Our goal is to build an enduring community of researchers who continue to learn from each other's methods and philosophies, continuing with impactful research contributions over the long term.

Two of the present organizers have been involved in producing a HDI-focused workshop at CHI '19 [7]. The 2019 workshop was a success, but limitations stemming from venue capacity, time limit, and thematic focus were observed. For 2020, we are aligning the theme towards diversity and interdisciplinarity, and planning for a full day of activities including intensive hands-on designing and prototyping. Furthermore, leveraging our involvements in HDI research projects within larger-scale clusters (see section: Organizers), we aim to expand participant recruitment efforts to vastly greater audiences including scholars of social sciences and humanities, autonomous systems and AI, architecture, and law.

\section{Organizers}

The workshop's international and multi-disciplinary team of organizers includes 6 researchers from Turkey, Sweden, Germany, France, and Australia.

Mehmet Aydın Baytaş received his PhD from Koç University's interdisciplinary Design, Technology and Society program. He is a Marie Skłodowska-Curie Research Fellow at Qualisys AB. His current research addresses the design space of autonomous drones operating in human-populated environments [2, 4].

Markus Funk is a senior user experience researcher at Cerence. Inc, Germany. Markus is an expert in humandrone interaction and flying user interfaces $[11,16]$.

Sara Ljungblad is an assistant professor at the University of Gothenburg and Chalmers University of Technology. She is interested in inclusive design methods and has done several long-term studies understand experiences of robotic products in everyday settings e.g. $[10,20]$. She is currently funded by WASP-HS ${ }^{1}$ to conduct constructive and critical design research on "social drones."

Jérémie Garcia is an assistant professor at ENAC, Toulouse, France. $\mathrm{He}$ is interested in designing interactions to author and operate automated systems including drones and air traffic control $[8,12]$.

Joseph La Delfa is a PhD student at the Exertion Games Lab, Monash University. His research $[17,18,19]$ is focused on bodily awareness and reflection through HDI (Fig-

${ }^{1}$ wasp-hs.org/projects/the-rise-of-social-drones-a-constructive-designresearch-agenda/

W34, Page 2 


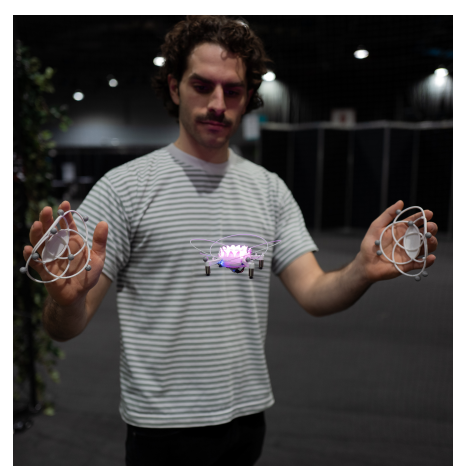

Figure 1: A novel Tai Chi-inspired meditative movement practice designed using a Crazyflie micro-quadcopter, augmented with floral cladding $[18,17]$. (Photo by Mehmet Aydın Baytaş) ure 1). His experiences in product design and engineering, from concept to production and maintenance, covers industries including glassware, tools, furniture, and service design.

Florian 'Floyd' Mueller is a professor at Monash University, where he directs the Exertion Games Lab. His research straddles the intersection of human-computer interaction, the body, and play; including the use of drones in exercise and recreation [17, 19, 23, 24].

Additionally, Bitcraze ${ }^{2}$ - makers of the Crazyflie open source micro-quadcopter platform - have confirmed their participation as an industry supporter. A number of Crazyflie drones and components will be provided to participants during the workshop (along with others supplied by the organizers) to support hands-on activities (flight subject to approval).

All inquiries can be addressed to the first author.

\section{Website}

The workshop website is: socialdrones.github.io/ihdi2020

The website promotes the workshop and presents relevant research results to date (both from organizers' own work, and from related work that we are aware of). All accepted contributions will appear on the website. Following the workshop itself, videos, photos, and results from workshop activities will be added.

In addition to the website, the organizers are maintaining the GitHub organization Social Drones, which is meant to be a hub for open resources and software that is relevant for the design and development of autonomous interactive drones: github.com/socialdrones

\footnotetext{
${ }^{2}$ bitcraze.io
}

\section{Pre-Workshop Plans}

We will publish a call for papers on the workshop website, and circulate it broadly to different individuals and communities who have interest in the workshop's subjects. This includes posting the call to mailing lists (e.g. chi-Announcements), posting on social media (e.g. Twitter, Facebook), and contacting researchers in our networks.

The principal format for submissions will be a paper in the $\mathrm{CHI}$ Extended Abstract format, up to 6 pages in length (including references). We welcome a diverse array of contributions, including empirical research, engineering investigations, pictorials (Figure 3), design concepts, theory, opinions, reviews, and works-in-progress. At least one author of each submission will be required to participate in the workshop and deliver a poster, demonstration, or presentation. No limitations will be imposed in terms of who can submit - submissions may be authored, for instance, by industry professionals and independent researchers.

We are expecting a diverse variety of topics and contributions to be covered, including but not limited to:

- Design proposals for novel experiences featuring both autonomous and piloted drones

- Theoretical, ethical and philosophical arguments that aim to promote clarity of thought and communication around HDI concepts and their use situations

- Inclusive design issues for HDI

- Transferring learnings between HDI and other $\mathrm{HCl} / \mathrm{HDI}$ topics or extracurricular disciplines

- Policy, law, and regulation around drones and HDI

- Tools and methods for all stages of HDI prototyping and development life cycles

- Fieldwork and ethnography (including visual) covering drone users, developers, and bystanders 


\section{Tentative Workshop Timeline}

\section{5 min Opening}

$15 \mathrm{~min}$ Introduction and ice-breaking

Session 1

20 min Keynote

$100 \mathrm{~min}$ Presentations and Posters

30 min Break

Session 2

120 min Prototyping Drone Issues

20 min Break

\section{Session 3}

40 min Presentations and Discussion

30 min Summary and Closing

Total: 6 h 30min

Afterwards Dinner and Networking
Submissions will be received and managed via EasyChair. Each submission will be reviewed by at least two of the workshop organizers. Acceptance will be based on quality and diversity. We expect to select around 10 papers each for both of the poster and demonstration/presentation tracks. Acceptance decisions will be sent to authors by February 28, 2020.

Submitting authors will also be polled for topics that they would like to see covered at the workshop, as well as for methods and tactics that they employ in their work. These will be consolidated and published on the website. This information will guide both organizers and participants in preparing for the workshop, particularly with respect to the hands-on activities.

All accepted papers will be made available through the workshop website. Participants will be expected to have read the papers before the workshop, and to arrive with relevant questions and discussion points.

Topics of interest, as well as research methods and tactics suggested by participants will be consolidated and published on the workshop website to guide the content and preparations. These topics will be linked to the workshop papers, and examples of other related work will be posted. We will also solicit and welcome suggestions from authors of accepted papers on various kinds of content that can be offered on the website before and after the workshop.

A formal application to the Hawai'i Convention Center will be submitted for permission to fly drones within the premises. (We note that while being able to fly drones is beneficial for the workshop, it is not critical; we will still be able to have a productive workshop without this particular activity.)

\section{Workshop Structure}

The workshop is planned to last for a full day. The tentative timeline is given on the sidebar.

The workshop will open with the organizers introducing themselves, as well as scope of the workshop. Each participant will then introduce themselves and their research in 30 seconds each. The main workshop activities will then take place over sessions with breaks in between.

\section{Session 1: Introduction and Research Presentations}

The bulk of the first session will be brief research presentations. The authors of each submission will have the option of delivering a presentation or preparing a poster. The session will also include at least one keynote presentation, delivered by a leading researcher who has realized significant contributions in relevant topics (TBA). Additional keynote speakers (including industry) will be scheduled based on the interests and contributions of attendees. The session will be planned to allow for questions and discussions.

\section{Session 2: Design and Prototyping}

The second session will concentrate on hands-on activities with participants working in groups of 3-5. (Ideally, groups will be formed during the preceding poster session and coffee break.) In line with the inclusive and integrative focus of the workshop, participants will be working on a gamut of design and prototyping activities that address human-drone interaction situations and related issues and questions. After, finalizing the groups, we will ask each group to quickly discuss and declare a particular subject matter and perspective/method. Groups will then be given time to sketch and prototype particular artifacts and experiences that pertain to human-drone interaction ecosystems, construed holistically. Although participants will be free to decide on a topic and methods, organizers will provide a menu of different subjects and techniques, as well as props, that may 


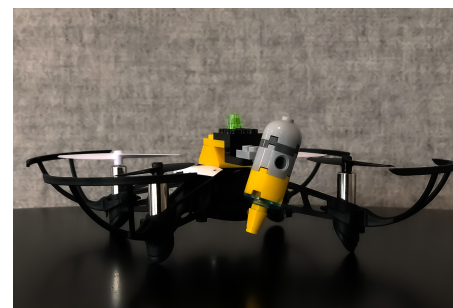

be addressed or used by the groups. This may include (but is not limited to) the following examples:

- Using video sketching $[21,27]$ to depict scenarios of living with a drone "pet" in a home environment

- Creating a working prototype - with a Crazyflie or other platform (flight subject to approval) - for a game that leverages proximity- and vision-based interactions

Figure 2: Using Lego to explore forms for a material deposition drone. (Photo by Mehmet Aydın Baytaş)

- Prototyping poster, booklet, etc. designs that communicate considerations for operating drones within a venue for pilots, programmers, and bystanders
- Designing controllers for pilots with diverse accessibility needs, via embodied sketching approaches [3, 22, 25, 28]

- Augmenting commodity drones with Lego or paper components to explore forms for various purposes (Figure 2)

- Drafting a script for a comedy on the bureaucratic hurdles experienced by the victim of an autonomous drone accident seeking compensation

We will provide various prototyping materials, as well as props such as drones, and photos of relevant contexts to stimulate reflection.

At the end of the activity, each group will have produced a concrete deliverable that they will present to the rest of the participants. The aim is to collectively cover a remarkably diverse set of topics and approaches, in order to introduce all participants to a rich variety of perspectives. The workshop organizers collectively have the gamut of expertise and experience that enables them to support participants in these activities; e.g. in mechanical engineering, software development, photography, industrial product design, creative writing, and graphic design. Participants are be encouraged to "visit" other groups during the activity, especially to solicit feedback on prototypes.
Session 3: Demonstrations and Discussion

The final session of the workshop will comprise presentations of and discussions on prototypes produced during the hands-on activity session. Participants are encouraged to demonstrate or enact, i.e. show rather than tell. The prototypes and presentations will be documented for possible future publication on the website and in other venues.

After a summary of the activities, organizers and participants will have time to discuss additional matters. We aim for a central discussion topic to be how we might continue as a community, e.g. identifying a knowledge sharing platform that fits into participants' existing practices.

\section{Post-Workshop Plans}

Workshop papers will be stored on the open archive $\mathrm{HAL}^{3}$ (indexed by Google Scholar). Papers will also be listed on the workshop website with links to the HAL archive.

Participants are invited to join for dinner (location TBA), continuing discussions and community-building.

A brief report on workshop activities, as well as selected photos and descriptions of outputs from the hands-on session (subject to creators' permissions) will be published on the workshop website. We also aim to explore other online platforms for continuous knowledge sharing, based on the preferences of the community.

A variety of post-workshop academic publications will be considered based on the content of the accepted submissions and the outputs of workshop sessions. These include but are not limited to: a report to be submitted to a venue that is relevant to the $\mathrm{CHI}$ community (e.g. ACM Interactions), full paper submissions to relevant SIGCHI confer-

\footnotetext{
${ }^{3}$ hal.archives-ouvertes.fr
} 


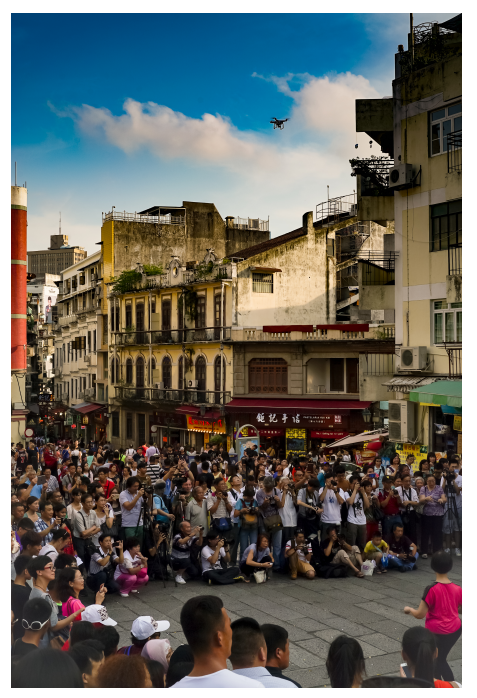

Figure 3: Photo by Eli Blevis [5] (used with permission), representing a photographic "construction of design knowledge" through a "carefully produced editorial record of a specific instance of a disruptive technology," documenting "the potential of drone photography to perturb the face to face social contract of the more conventional photography" [6]. Here, this image is meant to exemplify HDI scholarship via photography and storytelling. ences based on synergies between research efforts presented at the workshop, and scoping a special issue of a journal based on research agendas articulated at the workshop. (The editors-in-chief of ACM THRI have been contacted regarding an $\mathrm{HDI}$ special issue.)

\section{Call for Participation}

Drones are becoming ubiquitous. Current applications include logistics, construction, security, emergencies, and photography. Emerging applications such as exercise, companionship, and tangible user interfaces are active research topics within the $\mathrm{CHI}$ community.

Knowledge from a wealth of disciplines can inform new drone applications; and communication between different disciplines - design, engineering, social sciences, and humanities - is essential. To this end, the Interdisciplinary Workshop on Human-Drone Interaction (iHDI 2020) aims to intertwine diverse perspectives, as a platform for researchers and practitioners learning from each other.

We seek high quality contributions exploring HDI from a plethora of perspectives, including but not limited to: empirical research, engineering, design, theory, art, and opinions. Topics can include, but are not limited to, the following:

- Novel HDI experiences

- Theoretical and philosophical arguments

- Transferring learnings between $\mathrm{HDI}$ and other $\mathrm{HCl}$ or extracurricular topics

- Policy and regulatory issues

- Accessible HDI

- Prototyping and development tools

- Ethnographic fieldwork with users, developers, and bystanders
The submission deadline is 11 February 2020. Authors are invited to submit position papers, up to 6 pages (including references) in the $\mathrm{CHI}$ Extended Abstracts Format, at: easychair.org/conferences/?conf=ihdi2020

The proceedings will be stored on the open archive HAL (hal.archives-ouvertes.fr), indexed by Google Scholar. A paper on workshop outcomes will be submitted to a relevant venue. Selected contributions will be invited for publication in a special issue of the THRI journal.

At least one author must attend. Attendees must register for the workshop and at least one day of CHI 2020.

For more information: socialdrones.github.io/ihdi2020

\section{Acknowledgements}

The Exertion Games Lab appreciates and acknowledges the support from the School of Design at RMIT University.

\section{REFERENCES}

[1] Parastoo Abtahi, Benoit Landry, Jackie (Junrui) Yang, Marco Pavone, Sean Follmer, and James A. Landay. 2019. Beyond The Force: Using Quadcopters to Appropriate Objects and the Environment for Haptics in Virtual Reality. In Proceedings of the $2019 \mathrm{CHI}$ Conference on Human Factors in Computing Systems (CHI '19). ACM, New York, NY, USA, Article 359, 13 pages. DOI :

http://dx.doi.org/10.1145/3290605.3300589

[2] Mehmet Aydın Baytaş, Damla Çay, Yuchong Zhang, Mohammad Obaid, Asım Evren Yantaç, and Morten Fjeld. 2019. The Design of Social Drones: A Review of Studies on Autonomous Flyers in Inhabited Environments. In Proceedings of the 2019 CHI Conference on Human Factors in Computing Systems (CHI '19). ACM, New York, NY, USA, Article 250,13 pages. DOI :

http://dx.doi.org/10.1145/3290605.3300480

[3] Mehmet Aydın Baytaş, Yücel Yemez, and Oğuzhan Özcan. 2014. Hotspotizer: End-user Authoring of Mid-air Gestural Interactions. In Proceedings of the 8th Nordic Conference on Human-Computer Interaction: Fun, Fast, Foundational (NordiCHI '14). ACM, New York, 
NY, USA, 677-686. DOI :

http://dx.doi.org/10.1145/2639189.2639255

[4] Mehmet Aydın Baytaş, Mohammad Obaid, Joseph La Delfa,

Asım Evren Yantaç, and Morten Fjeld. 2019. Integrated Apparatus for Empirical Studies with Embodied Autonomous Social Drones. In 1st International Workshop on Human-Drone Interaction.

https://hal . archives-ouvertes .fr/hal-02128387

[5] Eli Blevis. 2015. Drones. interactions 22, 5 (Aug. 2015), 80-80. DOI : http://dx.doi.org/10.1145/2815099

[6] Eli Blevis. 2016. Being Photo-Visual in $\mathrm{HCl}$ and Design. In Proceedings of the 2016 ACM Conference on Designing Interactive Systems (DIS '16). ACM, New York, NY, USA, 983-995. DOI : http://dx.doi.org/10.1145/2901790.2901863

[7] Anke M. Brock, Jessica Cauchard, Markus Funk, Jérémie Garcia, Mohamed Khamis, and Matjaž Kljun. 2019. iHDI: International Workshop on Human-Drone Interaction. In Extended Abstracts of the 2019 CHI Conference on Human Factors in Computing Systems (CHI EA '19). ACM, New York, NY, USA, Article W01, 7 pages. DOI : http://dx.doi.org/10.1145/3290607.3299001

[8] Stéphane Conversy, Jeremie Garcia, Guilhem Buisan, Mathieu Cousy, Mathieu Poirier, Nicolas Saporito, Damiano Taurino, Giuseppe Frau, and Johan Debattista. 2018. Vizir: A Domain-Specific Graphical Language for Authoring and Operating Airport Automations. In Proceedings of the 31st Annual ACM Symposium on User Interface Software and Technology (UIST '18). ACM, New York, NY, USA, 261-273. DOI :

http://dx.doi.org/10.1145/3242587.3242623

[9] Sara Eriksson, Åsa Unander-Scharin, Vincent Trichon, Carl Unander-Scharin, Hedvig Kjellström, and Kristina Höök. 2019. Dancing With Drones: Crafting Novel Artistic Expressions Through Intercorporeality. In Proceedings of the $2019 \mathrm{CHI}$ Conference on Human Factors in Computing Systems (CHI '19). ACM, New York, NY, USA, Article 617, 12 pages. DOI :

http://dx.doi.org/10.1145/3290605.3300847

[10] Ylva Fernaeus, Maria Håkansson, Mattias Jacobsson, and Sara Ljungblad. 2010. How Do You Play with a Robotic Toy Animal?: A Long-term Study of Pleo. In Proceedings of the 9th International Conference on Interaction Design and Children (IDC '10). ACM, New York, NY, USA, 39-48. DOI :

http://dx.doi.org/10.1145/1810543.1810549
[11] Markus Funk. 2018. Human-drone Interaction: Let's Get Ready for Flying User Interfaces! Interactions 25, 3 (April 2018), 78-81. DOI : http://dx.doi.org/10.1145/3194317

[12] Jérémie Garcia, Luc Chevrier, Yannick Jestin, and Anke M. Brock. 2019. HandiFly: Towards Interactions to Support Drone Pilots with Disabilities. In Extended Abstracts of the $2019 \mathrm{CHI}$ Conference on Human Factors in Computing Systems (CHI EA '19). ACM, New York, NY, USA, Article LBW0116, 6 pages. DOI :

http://dx.doi.org/10.1145/3290607.3312957

[13] Antonio Gomes, Calvin Rubens, Sean Braley, and Roel Vertegaal. 2016. BitDrones. Interactions 23, 3 (April 2016), 14-15. DOI : http://dx.doi.org/10.1145/2898173

[14] Md. Nafiz Hasan Khan and Carman Neustaedter. 2019. An Exploratory Study of the Use of Drones for Assisting Firefighters During Emergency Situations. In Proceedings of the 2019 CHI Conference on Human Factors in Computing Systems (CHI '19). ACM, New York, NY, USA, Article 272, 14 pages. DOI : http://dx.doi.org/10.1145/3290605.3300502

[15] Pascal Knierim, Thomas Kosch, Alexander Achberger, and Markus Funk. 2018a. Flyables: Exploring 3D Interaction Spaces for Levitating Tangibles. In Proceedings of the Twelfth International Conference on Tangible, Embedded, and Embodied Interaction (TEI '18). ACM, New York, NY, USA. DOI :

http://dx.doi.org/10.1145/3173225.3173273

[16] Pascal Knierim, Steffen Maurer, Katrin Wolf, and Markus Funk. 2018b. Quadcopter-Projected In-Situ Navigation Cues for Improved Location Awareness. In Proceedings of the $2018 \mathrm{CHI}$ Conference on Human Factors in Computing Systems (CHI '18). ACM, New York, NY, USA, Article 433, 6 pages. DOI : http://dx.doi.org/10.1145/3173574.3174007

[17] Joseph La Delfa, Mehmet Aydın Baytaş, Olivia Wichtowski, Rohit Ashok Khot, and Florian "Floyd" Mueller. 2019. Are Drones Meditative? In Extended Abstracts of the $2019 \mathrm{CHI}$ Conference on Human Factors in Computing Systems (CHI EA '19). ACM, New York, NY, USA, Article INT046, 4 pages. DOI : http://dx.doi.org/10.1145/3290607.3313274

[18] Joseph La Delfa, Mehmet Aydın Baytaş, Rakesh Patibanda, Hazel Ngari, Rohit Ashok Khot, and Florian "Floyd" Mueller. 2020. Drone Chi: Somaesthetic Human-Drone Interaction. In Proceedings of the 2020 CHI Conference on Human Factors in Computing Systems (CHI '20). ACM, New York, NY, USA. 
[19] Joseph La Delfa, Robert Jarvis, Rohit Ashok Khot, and Florian 'Floyd' Mueller. 2018. Tai Chi In The Clouds: Using Micro UAV's To Support Tai Chi Practice. In Proceedings of the 2018 Annual Symposium on Computer-Human Interaction in Play Companion Extended Abstracts (CHI PLAY '18 EA). ACM, New York, NY, USA, 513-519. DOI : http://dx.doi.org/10.1145/3270316.3271511

[20] Sara Ljungblad, Jirina Kotrbova, Mattias Jacobsson, Henriette Cramer, and Karol Niechwiadowicz. 2012. Hospital Robot at Work: Something Alien or an Intelligent Colleague?. In Proceedings of the ACM 2012 Conference on Computer Supported Cooperative Work (CSCW'12). ACM, New York, NY, USA, 177-186. DOI : http://dx.doi.org/10.1145/2145204.2145233

[21] Jonas Löwgren. 2004. Animated Use Sketches As Design Representations. Interactions 11, 6 (Nov. 2004), 22-27. DOI : http://dx.doi.org/10.1145/1029036.1029048

[22] Elena Márquez Segura, Laia Turmo Vidal, Asreen Rostami, and Annika Waern. 2016. Embodied Sketching. In Proceedings of the 2016 CHI Conference on Human Factors in Computing Systems (CHI 16). ACM, New York, NY, USA, 6014-6027. DOI

http://dx.doi.org/10.1145/2858036.2858486

[23] Florian Mueller and Matthew Muirhead. 2014. Understanding the Design of a Flying Jogging Companion. In Proceedings of the Adjunct Publication of the 27th Annual ACM Symposium on User Interface Software and Technology (UIST'14 Adjunct). ACM, New York, NY, USA, 81-82. DOI :

http://dx.doi.org/10.1145/2658779.2658786
[24] Florian 'Floyd' Mueller and Matthew Muirhead. 2015. Jogging with a Quadcopter. In Proceedings of the 33rd Annual ACM Conference on Human Factors in Computing Systems (CHI '15). ACM, New York, NY, USA, 2023-2032. DOI :

http://dx.doi.org/10.1145/2702123.2702472

[25] Antti Oulasvirta, Esko Kurvinen, and Tomi Kankainen. 2003. Understanding contexts by being there: case studies in bodystorming. Personal and Ubiquitous Computing 7, 2 (01 Jul 2003), 125-134. DOI :

http://dx.doi.org/10.1007/s00779-003-0238-7

[26] Wataru Yamada, Hiroyuki Manabe, and Daizo Ikeda. 2019. ZeRONE: Safety Drone with Blade-Free Propulsion. In Proceedings of the 2019 CHI Conference on Human Factors in Computing Systems (CHI '19). ACM, New York, NY, USA, Article 365, 8 pages. DOI http://dx.doi.org/10.1145/3290605.3300595

[27] J. Zimmerman. 2005. Video sketches: exploring pervasive computing interaction designs. IEEE Pervasive Computing 4, 4 (Oct 2005), 91-94. DOI:http://dx.doi .org/10.1109/MPRV.2005.91

[28] Adviye Ayça Ünlüer, Mehmet Aydın Baytaş, Oğuz Turan Buruk, Zeynep Cemalcilar, Yücel Yemez, and Oğuzhan Özcan. 2018. The Effectiveness of Mime-Based Creative Drama Education for Exploring Gesture-Based User Interfaces. International Journal of Art \& Design Education 37, 3 (2018), 353-366. DOI :

http://dx.doi.org/10.1111/jade.12136 